\title{
Prinzipien der Frakturbehandlung beim Kind
}

Francisco F. Fernandez, Oliver Eberhardt

\section{Einleitung}

Warum sollten bei Kindern andere Behandlungsprinzipien gelten als beim Erwachsenen? Der viel zitierte Satz, dass „Kinder keine kleinen Erwachsenen sind“ hat seine Berechtigung aus den unterschiedlichsten Gründen. Kinder haben häufiger Frakturen als Erwachsene, sie sind meist schlechter in der Lage, Risiken adäquat einzuschätzen. Zudem sind Charakteristika des wachsenden Knochens wie hohe Flexibilität und geringere Stabilität zu berücksichtigen, die zu einer höheren Inzidenz an knöchernen Verletzungen führen.

Die besonderen Eigenschaften der Frakturheilung am wachsenden Skelett sind bekannt $[1,8,14]$. Das Skelett stellt ein vom Alter abhängiges, dynamisch wachsendes Organ dar mit der Fähigkeit, schnell knöcherne Verletzungen zu heilen, mit einer hohen Toleranz gegenüber Weichteilverletzung, geringen Immobilitätsschäden und der altersabhängigen Korrekturfähigkeit für posttraumatische Fehlstellungen [1,8]. Für eine kindgerechte Frakturbehandlung bedarf es spezieller Kenntnisse der Wachstumseigenschaften gesunder und verletzter Knochen sowie der Röntgenanatomie in den Altersgruppen.

Das Wissen über die Wachstumsphänomene und die Chance zur spontanen Korrektur sollte in den Behandlungsalgorithmus einbezogen werden.

Im Einzelnen soll hier auf den Unfallhergang, Diagnostik, Klassifikation, Wachstumsprognose, Spontankorrekturpotenzial und Therapieoptionen eingegangen werden.

\section{Unfallhergang}

Im ersten Schritt sollte geklärte werden, ob ein adäquates Ereignis mit entsprechendem Unfallmechanismus zur Fraktur geführt hat. Die Abgrenzung zwischen adäquat und inadäquat kann durchaus schwierig sein, denn hier spielt das Alter bereits eine große Rolle. Kleinkinder können durch ein bloßes Hinfallen bereits eine Fraktur der Tibia erleiden im Sinne einer „Toddler“-Fraktur, Kinder unter dem 6. Lebensjahr können durch Bagatellstürze traumatische Hüftluxationen erleiden oder Bagatellbelastungen können bei Kindern unter dem 10. Lebensjahr zu Kalkaneusfrakturen führen ( $\triangleright$ Abb. 1). Die Beurteilung des Unfallherganges und des Unfallmechanismus kann
Aufschluss und Hinweise geben über mögliche Knochenveränderungen bzw. andere Verletzungsursachen (Osteogenesis imperfecta, pathologische Fraktur, Kindesmisshandlung). Im Kindes- und Jugendalter können Knochenläsionen wie juvenile Knochenzysten, fibröse Dysplasien und andere Läsionen sich erstmalig mit einem Knochenbruch manifestieren ( $\bullet$ Abb. 2). Die Dunkelziffer von primär nicht festgestellten Frakturen kann sehr hoch sein, z. B. Kuboidfrakturen oder Kalkaneusfrakturen. Aufgrund einer nur sehr geringen Weichteilschwellung und häufig unbedeutendem Unfallmechanismus werden diese in bis zu $50 \%$ nicht erkannt [1].

Besonderes Augenmerk besteht bei zeitlicher Verzögerung zwischen Verletzungszeitpunkt und Vorstellung beim Arzt, wechselnden, unklaren oder widersprüchlichen Erklärungen zur Verletzungsursache, wenn der beschriebene Verletzungsmechanismus in keiner Weise zur Verletzung passt oder bei Schaftfrakturen bei Säuglingen, die noch nicht laufen können. Hier muss zwingend an eine Kindesmisshandlung gedacht werden. Dabei sind typische Verletzungsmuster eine Vielzahl unterschiedlicher Läsionen in unterschiedlichem Heilungsstadium, atypische Lokalisationen und Verletzungen in unterschiedlichen Körperregionen [5, 11].

\section{Untersuchung und Diagnostik}

Besonders bei Erstkontakt sollte sich die klinische Untersuchung zunächst auf die visuelle und verbale Kontaktaufnahme beschränken, die Inspektion gibt bereits deutliche Hinweise, wo das Kind seine Verletzung haben könnte. Die Untersuchung nach primären Frakturzeichen wie Knochenkrepitation oder abnorme Beweglichkeit sollte primär unterbleiben, sie fügt dem Kind unnötige Schmerzen zu. Nach einer Bildgebung ohne richtungsweisenden Befund kann eine Wiederholung sinnvoll sein, um dann die weitere Diagnostik zielgerichteter durchführen zu können.

Die Interpretation des Röntgenbildes kann durchaus schwierig sein. Durch das Wachstum ändern sich auf den konventionellen Röntgenbildern die knöchernen Strukturen ständig. Die Knochenkerne kommen zu unterschiedlichen Zeitpunkten und verschmelzen dann, insbesondere am Ellenbogen, bzw. es existiert eine Vielzahl an ak- 

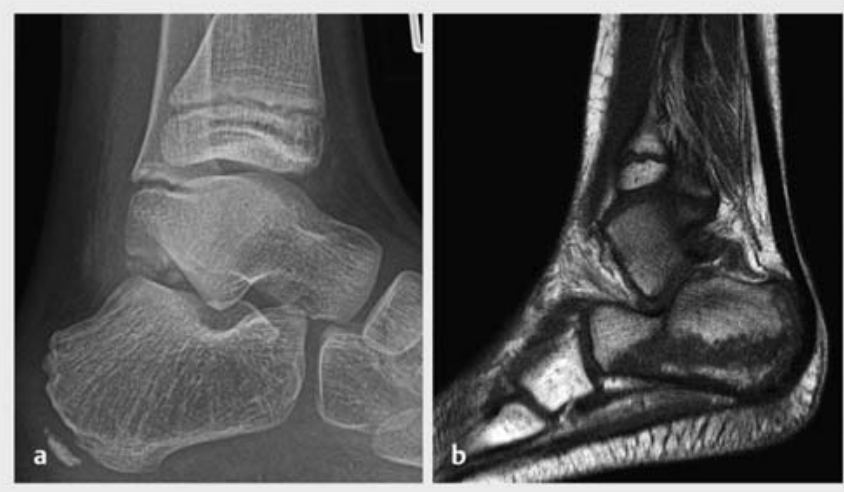

- Abb. 1 Zehnjähriges Mädchen mit Bagatellstauchungstrauma und geringer Schwellung des Rückfußes. Radiologisch kein Hinweis für eine Kalkaneusfraktur. Im NMR-Bild extraartikuläre nicht dislozierte Kalkaneusfraktur.

zessorischen Kernen, was die Beurteilung erheblich erschwert.

Bei Frakturen muss der neurologische Status und Gefäßstatus untersucht und dokumentiert werden. Es kann beim Kind durchaus schwierig sein, einen neurologischen Status zu erheben, mitunter braucht es Geduld und Zeit. Postoperative neurologische Defizite sind insbesondere dann eine besondere Herausforderung, wenn bei der primären Untersuchung neurologische Defizite nicht ausgeschlossen wurden, wie z.B. bei suprakondylären Frakturen.

In der Kindertraumatologie kommen die Sonografie, das konventionelle Röntgen, Computertomografie und Magnetresonanztomografie zum Einsatz $[1,8,14]$. Nach wie vor stellt aber das konventionelle Röntgen das Hauptdiagnostikum dar. Prinzipiell sollten Röntgenaufnahmen in 2 Ebenen mit Abbildung der angrenzenden Gelenke angefertigt werden, ist jedoch in einer Ebene die Indikation zur Operation gestellt worden, sollte auf die 2. Ebene verzichtet werden ( $\boldsymbol{A}$ Abb. 3). Ein Verzicht auf die Darstellung des Ellenbogengelenkes bei einer Ulna- oder Unterarmfraktur kann dazu führen, dass die offensichtliche Ulnaschaftfraktur gesehen und behandelt wird, aber der luxierte Radiuskopf nicht zur Darstellung kommt und damit übersehen wird. Damit nimmt eine mögliche Prognose dieser übersehenen Monteggia-Läsion ihren unheilvollen Lauf. Die Sonografie hat ihren Stellenwert bei Strukturen, die noch knorpelig angelegt sind, wie z.B. der Radiuskopf, und es können mit ihrer Hilfe zuverlässig Wulst- und Stauchungsfrakturen diagnostiziert werden. Computertomografie und Magnetresonanztomografie (NMR) haben in der Akutdiagnostik an den Extremitäten einen untergeordneten Stellenwert, nicht so bei der Diagnostik von Wirbelsäulenverletzungen. Auch bei Gelenkläsionen, insbesondere am Kniegelenk, hat das NMR

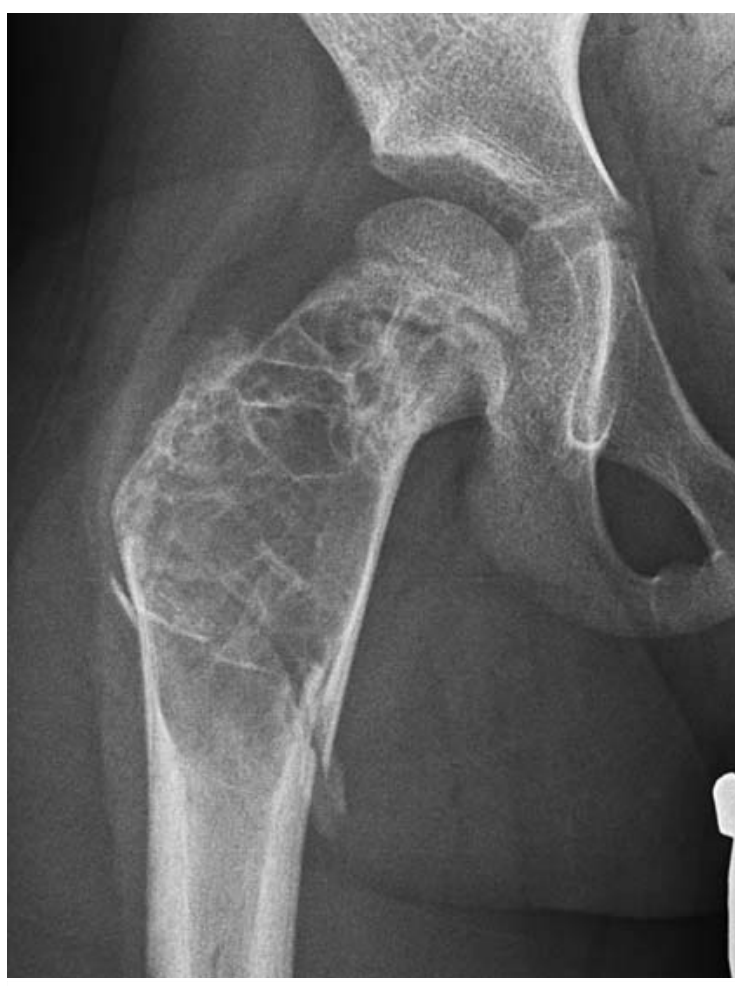

- Abb. 2 Sechsjähriger Junge mit pathologischer subtrochantärer Femurfraktur ohne adäquates Trauma. Bis zu diesen Bruch keine Beschwerden an der Hüfte gehabt.

eine zunehmende Bedeutung. Wenn keine Fraktur erkennbar ist, so kann das NMR ligamentäre Verletzungen wie vordere Kreuzbandrupturen oder bei Patellaluxationen Begleitverletzungen wie osteochondrale Flakes nachweisen.

\section{Wachstumsphänomene, Spontan- korrektur und Wachstumsstörungen}

Die Entstehung von Knochenwachstum ist bekannt [1,8, 14]. Das Dickenwachstum findet durch periostalen Knochenanbau und gleichzeitigen endostalen Knochenabbau statt. Der Knochen folgt dabei dem von Roux formulierten Gesetz: mit einem Minimum an Material ein Optimum an Belastbarkeit zu erreichen. Für das Längenwachstum ist die Wachstumsfuge in unterschiedlichem Ausmaß, je nach Lokalisation und Alter, verantwortlich ( $\vee$ Abb. 4). Die regionale spontane Korrektur von posttraumatischen Fehlstellungen ist abhängig von der Wachstumsbeteiligung der angrenzenden Fuge an der Gesamtlänge des Knochens. Im Bereich der oberen Extremität ist die Wachstumsbeteiligung an der proximalen Humerusepiphyse und an der distalen Radiusepiphyse mit ca. 80 \% für die Gesamtlänge verantwortlich [1,8,14]. Im Bereich der unteren Extremität erfolgt anteilsmäßig das größte Längenwachstum um das Kniegelenk herum 


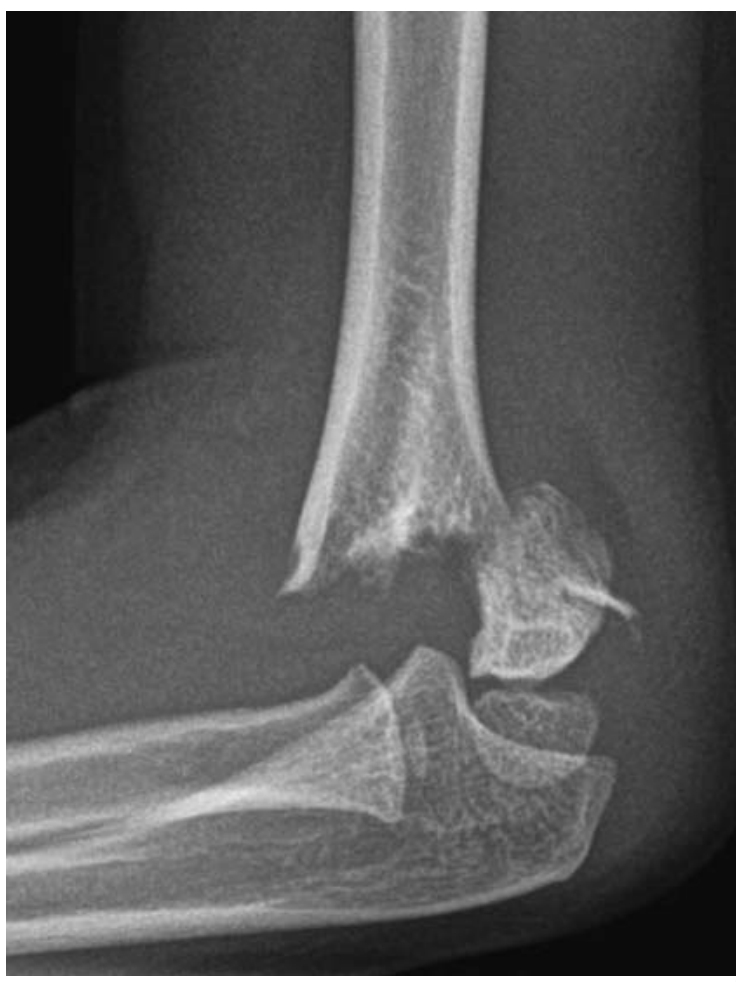

- Abb. 3 Grob dislozierte suprakondyläre Humerusfraktur Typ IV nach AO. Indikation zur Operation ist eindeutig gegeben, weitere Röntgenaufnahmen führen zu Beschwerden ohne Änderung der Indikation.

mit der distalen Femurepiphyse bzw. der proximalen Tibiaepiphyse von ca. 60\%. Achsknicke an diesen hochprozentigen Wachstumsfugen werden erheblich besser korrigiert als bei niedrigprozentigen.

Die Spontankorrektur ist jedoch auch von der funktionellen Beanspruchung abhängig, so werden Achsabweichungen in der Hauptbewegungsrichtung positiv beeinflusst [1].

Die spontanen Korrekturmechanismen von Achsabweichung und Seit-zu-Seit-Verschiebung kann der Knochen durch Knochenabbau auf der Zugseite und Knochenanbau auf der Druckseite remodellieren. Inwieweit sich die physiologischen Rotationsvorgänge auf einen Rotationsfehler auswirken, ist nicht abschließend beurteilbar, wenn, dann laufen sie ungezielt ab. Die Spontankorrekturpotenz und das Risiko für Wachstumsstörungen treten nur bei ausreichend verbleibender Zeit für das Restwachstum auf und hängen ab von: Alter, Entwicklungsstadium, Geschlecht, Dislokationsrichtung- und -ausmaß $[1,8,14]$.

Bei der Planung der Behandlungsstrategie muss abgeschätzt werden, ob man das Potenzial der Wachstumsfuge in die Therapie mit einbaut. Eine hochpotente Fuge erhöht das Potenzial zur Spontankorrektur posttraumati-

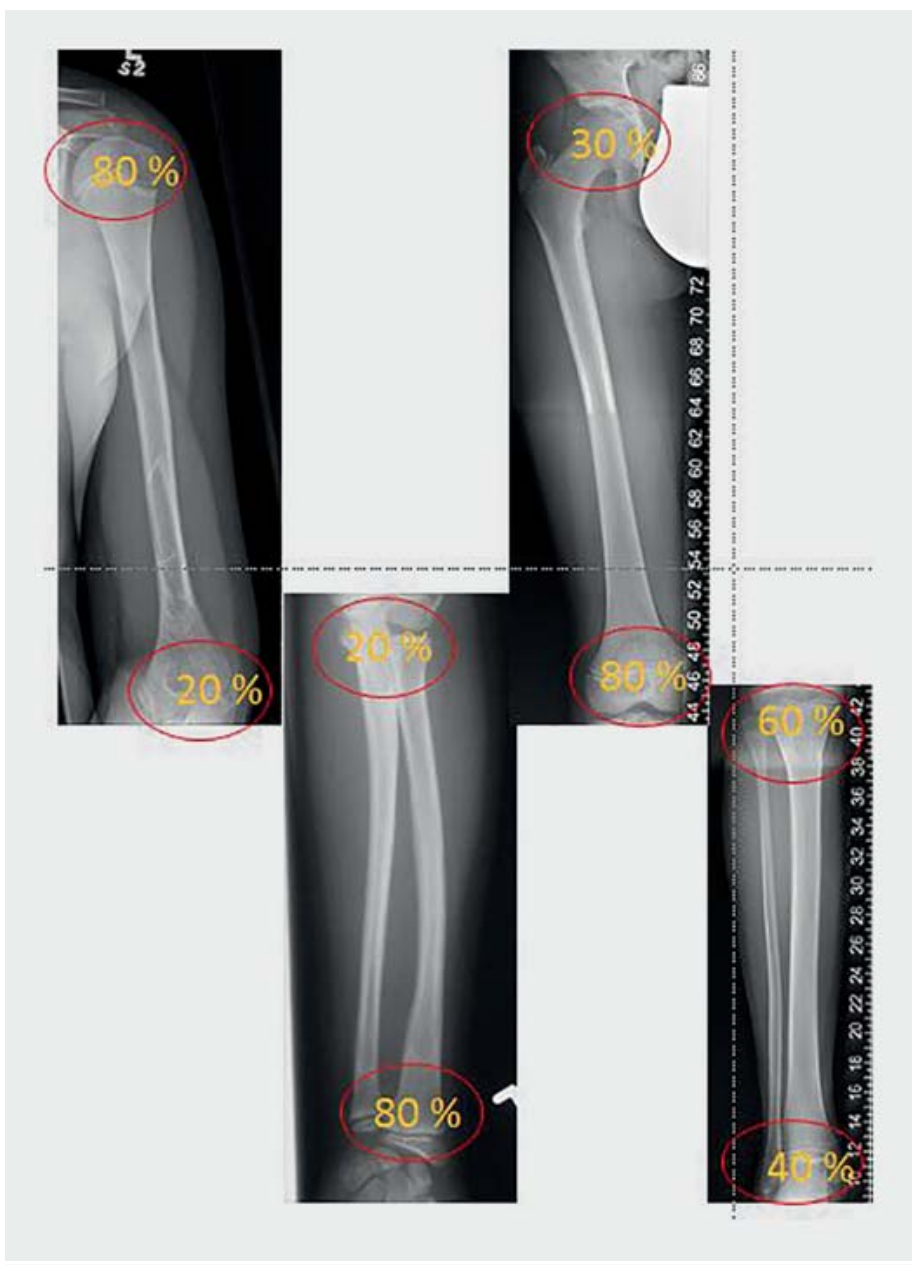

- Abb. 4 An den Enden der 4 langen Röhrenknochen ist je eine Epiphysenfuge. Der Anteil der einzelnen Fugen am Längenwachstum der einzelnen Röhrenknochen ist sehr unterschiedlich.

scher Fehlstellungen, aber gleichermaßen auch die Anfälligkeit gegenüber Wachstumsstörungen. Wird eine Fehlstellung kalkuliert belassen, so müssen besondere Kenntnisse zur Abschätzung der Remodellierung vorliegen.

\section{Behandlungsprinzipien}

In der Primärversorgung des verletzten Kindes sollte das erste Therapieziel die Schmerzfreiheit sein. Noch vor Beginn von diagnostischen Maßnahmen sollte nach der Inspektion eine provisorische Ruhigstellung durchgeführt und bei Bedarf mit Analgetika kombiniert werden. Nach der Diagnostik muss festgelegt werden, welcher Behandlungsstrategie man folgt. Ungeplante Verfahrenswechsel und Wiederholungseingriffe sollten möglichst unterbleiben, außer, sie werden gezielt in die Therapiestrategie mit eingeplant. Schmerzhafte Manipulationen wie Reposition, Redression und redressierende Verbände sollten in einer adäquaten Analgosedierung oder Narkose durchgeführt werden. Liegt jedoch ein Kind in Narkose, sollte eine definitive Versorgung angestrebt werden. Problema- 

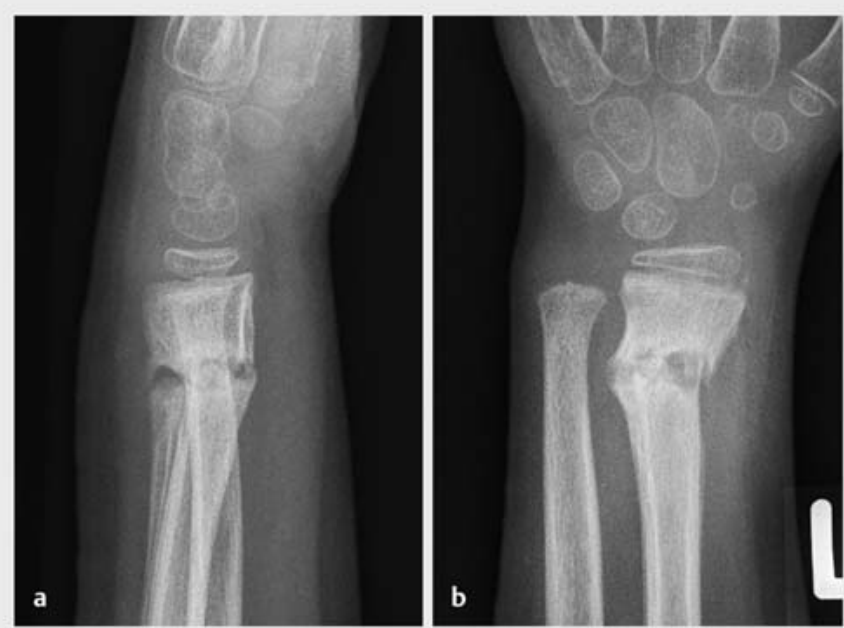

- Abb. 5 a, b Siebenjähriger Junge nach offener Reposition und perkutaner K-Draht-Osteosynthese einer distalen Radiusfraktur. Nach 4 Monaten noch keine ausreichende knöcherne Konsolidierung und drohende Pseudarthrose.

tisch ist diese Philosophie, da bei Kleinkindern und jüngeren Kindern dann eventuell eine Ostosynthese erfolgt, nur um einen Korrekturverlust zu vermeiden, was einem „Overtreatment“ entsprechen kann. Dabei muss berücksichtigt werden, dass sich ein Korrekturverlust bei einem Kleinkind durch die Remodellierung ohne operative Korrektur vollständig zurückbilden kann.

In einzelnen Fällen führt diese Strategie der sofortigen definitiven Versorgung zu einer operativen Versorgung mit bedeutenden Komplikationen wie verzögerter Knochenbruchheilung bis hin zu Pseudarthrosen ( $>$ Abb. 5),
Infektverläufen und Osteomyelitis, was bei einer konservativen Behandlung so gut wie nicht auftreten würde [13].

\section{Therapiemethoden}

An Therapieoptionen stehen konservative und operative Verfahren zur Verfügung. Die Behandlungsstrategie hängt vom Alter, Ausmaß der Frakturdislokation und der Stabilität, der Frakturlokalisation und dem bestehenden Weichteilschaden ab.

In den kommenden folgenden Beiträgen wird gesondert auf die jeweiligen Frakturen an den Extremitäten eingegangen. Von daher ist es nicht das Ziel, in diesem Beitrag spezielle Fragestellungen vorwegzunehmen.

Vorweggenommen werden soll, dass es in der Kinderund Jugendtraumatologie in den letzten 20 Jahren zu einer massiven Erweiterung der operativen Indikationen gekommen ist. Insbesondere hat die elastische stabile intramedulläre Nagelung (ESIN) quasi zu einer „Revolution“ in der Versorgung von Schaftfrakturen geführt [2, 7,9]. Allerdings hat diese Technik durch ihre sehr guten Ergebnisse auch dazu geführt, dass die Fähigkeiten der Kliniken zur konservativen Behandlung abgenommen haben. Die Grenzen und Komplikationsmöglichkeiten der ESIN sind zwischenzeitlich bekannt $[3,10,12]$. Unter anderem kam es zu einer Ausweitung der Operationsindikationen auf Frakturen, z. B. Oberschenkelschaftfrakturen ( $\triangleright$ Abb. 6) bei Kleinkindern, die konservativ sehr gut behandelbar sind [4].

Im Allgemeinen werden undislozierte oder akzeptabel dislozierte Frakturen konservativ behandelt [6]. Zur lokalisations- und altersabhängig akzeptablen Dislokation sei
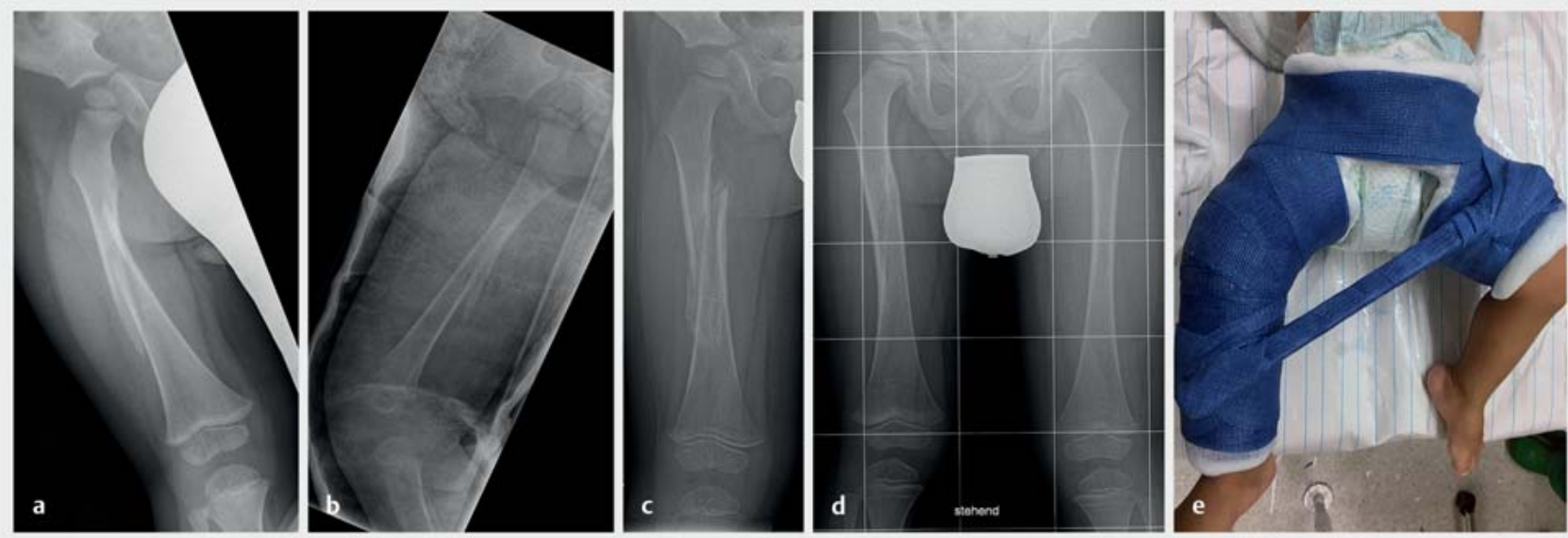

- Abb. 6 a-d 3-jähriger Junge mit Oberschenkelspiralfraktur. Versorgung mittels Beckenbeingips für 3 Wochen. Nach 3 Wochen gute Kallusbildung mit Verkürzung um ca. $1,5 \mathrm{~cm}$. Bei der Verlaufskontrolle 14 Monate später zeigte sich ein um wenige Millimeter größeres Femur. 
auf die Literatur verwiesen $[1,6,8,14]$. Akzeptabel ist die konservative Therapie dann, wenn eine verbliebene Dislokation durch das weitere Wachstum remodelliert werden kann und hieraus kein Bewegungsdefizit entsteht. Man muss sich aber bewusst darüber sein, dass gewisse Fehlstellungen zwar vollständig remodellieren, aber ein Bewegungsdefizit verbleibt, z. B. können Unterarmschaftfrakturen in einer Fehlstellung vollständig remodellieren, das Unterarmumwendbewegungsdefizit kann aber verbleiben.

Die konservative Therapie von Frakturen bedarf der gleichen Hingabe und des gleichen Aufwandes wie eine operative Therapie, um ebenfalls ein gutes Ergebnis zu erzielen. Von der Redression bis hin zum retendierenden Verband muss auch hier die Therapiekette angepasst werden.

Ist eine Reposition notwendig, muss durch eine redressierende Ruhigstellung versucht werden, eine Redislokation zu verhindern. Wenn dies allein durch eine Immobilisation nicht möglich ist, dann muss über eine operative Stabilisierung nachgedacht werden, hier kommt es wieder besonders auf das Alter an.

Folgende Osteosynthesen kommen zur Anwendung, die stark von der Frakturlokalisation abhängig sind: Bei dislozierten epiphysären Frakturen kommen offene oder geschlossene Repositionen zur Anwendung [1,8]. Das Ziel bei Gelenkfrakturen sollte es sein, die Gelenkflächen stufenlos zu readaptieren. Die Gelenkflächenrekonstruktion kann durch Kirschner-Drähte oder Kompressionsschrauben durchgeführt werden. Platten eignen sich nur begrenzt dazu, sie würden zu einer Epiphyseodese führen. Plattenosteosynthesen können dann sinnvoll sein, wenn die Wachstumsfuge kurz vor dem Verschließen ist, z. B. bei einer intraartikulären Tuberositas-tibiae-Fraktur mit weit vorgeschrittenem Wachstumsfugenverschluss. Unter arthroskopischer Repositionskontrolle können auch Gelenkfrakturen stufenlos versorgt werden.

Metaphysäre Frakturen sind meist gut geschlossen reponierbar, aber häufig nicht redressierbar, sodass oft eine Fixation erforderlich wird. Diese kann mit KirschnerDrähten oder Schrauben stabilisiert werden. Häufig bedarf es noch einer zusätzlichen Ruhigstellung. Eine intramedulläre Schienung kann hier ebenfalls erfolgreich sein, das Wirkungsprinzip entspricht jedoch dann nicht mehr der elastisch stabilen intramedullären Nagelung (ESIN). Plattenosteosynthesen im Bereich der Metaphyse bei Kindern sind eine Seltenheit und kommen eher bei Jugendlichen zur Anwendung. Diaphysäre Frakturen werden in Abhängigkeit vom gebrochenen Knochen sehr unterschiedlich versorgt. Die ESIN hat die Versorgung der Schaftfrakturen revolutionär verändert $[2,7,9,10]$. Hier gibt es sehr gute Indikationen und auch solche, in denen diese Technik an ihre Grenzen gebracht wird und dann zu fraglichen Ergebnissen kommt [10]. Wir möchten hier die Versorgung nicht weiter vertiefen, dies wird in den folgenden Beiträgen noch spezieller beleuchtet werden. Plattenosteosynthesen werden nur in wenigen Fällen als durchgeschobene Platten, z. B. bei gelenknahen Frakturen bei Adoleszenten, angewendet. Der Einsatz von Verriegelungsnägeln wird im Wesentlichen bei diaphysären Frakturen bei Jugendlichen vor dem Wachstumsabschluss oder z. B. bei Kindern über $50 \mathrm{~kg}$ am Oberschenkel eingesetzt.

Postoperative Röntgenkontrollen sollten auf ein Minimum reduziert werden.

Die Metallentfernung sollte, insbesondere bei der ESIN, nicht zu früh durchgeführt werden, um nicht Refrakturen zu provozieren.

\section{Interessenkonflikt}

Von Seiten der Autoren bestehen keine Interessenkonflikte.

\section{Autorinnen/Autoren}

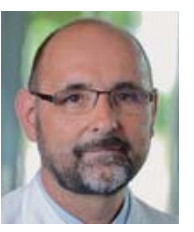

\section{Francisco F. Fernandez}

PD Dr. med., Ärztlicher Leiter der Kinder- und Jugendtraumatologie, Olgahospital/Klinikum Stuttgart

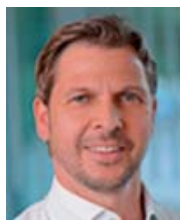

\section{Oliver Eberhardt}

PD Dr. med., Oberarzt, Orthopädische Klinik, Olgahospital/Klinikum Stuttgart

\section{Korrespondenzadresse}

PD Dr. med. Francisco F. Fernandez

Kinder- und Jugendtraumatologie Olgahospital/Klinikum Stuttgart

Kriegsbergstraße 62

70174 Stuttgart

Tel.: 0711/27873017

f.fernandez@klinikum-stuttgart.de

\section{Literatur}

[1] Beaty JH, Kasser JR, eds. Rockwood and Wilkins' Fractures in Children. 6th ed. Philadelphia: Lippincott Williams \& Wilkins

[2] Fernandez FF, Eberhardt O, Wirth T. [Elastic stable intramedullary nailing as alternative therapy for the management of paediatric humeral shaft fractures]. Z Orthop Unfall 2010; 148: 49-53

[3] Fernandez FF, Eberhardt O, Langendörfer $\mathrm{M}$ et al. Nonunion of forearm shaft fractures in children after intramedullary nailing. J Pediatr Orthop B 2009; 18: 289-295 
[4] Heffernan M], Gordon JE, Sabatini CS et al. Treatment of femur fractures in young children: a multicenter comparison of flexible intramedullary nails to spica casting in young children aged 2 to 6 years. J Pediatr Orthop 2015; 35: 126-129

[5] Kemp AM, Dunstan F, Harrison S et al. Patterns of skeletal fractures in child abuse: systematic review. BMJ 2008; 337: a1518. doi:10.1136/bmj.a1518

[6] Kraus R, Wessel L. The treatment of upper limb fractures in children and adolescents. Dtsch Arztebl Int 2010; 107: 903910

[7] Ligier JN, Métaizeau JP, Prévot J et al. Elastic stable intramedullary pinning of long bone shaft fractures in children. Z Kinderchir 1985; 40: 209-212

[8] Marzi I, Hrsg. Kindertraumatologie. 2. Aufl. Berlin, Heidelberg: Springer; 2009

[9] Prévot ], Lascombes P, Ligier ]N. [The ECMES (centro-medullary elastic stabilizing wiring) osteosynthesis method in limb fractures in children. Principle, application on the femur. Apropos of 250 fractures followed- up since 1979]. Chirurgie 1993; 119: $473-476$
[10] Schmittenbecher PP. Complications and errors in use of intramedullary nailing in shaft fractures in childhood. Kongressbd Dtsch Ges Chir Kongr 2001; 118: 435-437

[11] Skellern CY, Wood DO, Murphy A et al. Non-accidental fractures in infants: risk of further abuse. J Paediatr Child Health 2000; 36: 590-592

[12] Slongo T. Complications and failures of the ESIN technique. Injury 2005; 36 (Suppl. 1): A78-A85

[13] Subramanian P, Kantharuban S, Shilston S et al. Complications of Kirschner-wire fixation in distal radius fractures. Tech Hand Up Extrem Surg 2012; 16: 120-123

[14] von Laer L, Kraus R, Linhart WE, Hrsg. Frakturen und Luxationen im Wachstumsalter. 6. Aufl. Stuttgart: Thieme; 2012

\section{Bibliografie}

DOI https://doi.org/10.1055/a-0607-8097

OP-JOURNAL 2018; 34: 201-206 @ Georg Thieme Verlag KG Stuttgart · New York ISSN 0178-1715 